\title{
Emergence of Coordinated Plasticity in the Cochlear Nucleus and Cerebellum
}

\author{
Hua Yang and Matthew A. Xu-Friedman \\ Department of Biological Sciences, University at Buffalo, State University of New York, Buffalo, New York 14260
}

\begin{abstract}
Synapses formed by one cell type onto another cell type tend to show characteristic short-term plasticity, which varies from facilitating to depressing depending on the particular system. Within a population of synapses, plasticity can also be variable, and it is unknown how this plasticity is determined on a cell-by-cell level. We have investigated this in the mouse cochlear nucleus, where auditory nerve (AN) fibers contact bushy cells (BCs) at synapses called "endbulbs of Held." Synapses formed by different AN fibers onto one BC had plasticity that was more similar than would be expected at random. Experiments using MK-801 indicated that this resulted in part from similarity in the presynaptic probability of release. The similarity was not present in immature synapses but emerged after the onset of hearing. In addition, the phenomenon occurred at excitatory synapses in the cerebellum. This indicates that postsynaptic cells coordinate the plasticity of their inputs, which suggests that plasticity is of fundamental importance to synaptic function.
\end{abstract}

\section{Introduction}

Different synapses in the nervous system commonly exhibit different forms of short-term synaptic plasticity. This is observed even when one neuron makes synapses onto multiple target cell types, a phenomenon referred to as target cell-specific synaptic plasticity (Markram et al., 1998; Reyes et al., 1998; Rozov et al., 2001). Synaptic plasticity may provide different computational characteristics to synapses. For example, depression has been proposed to make postsynaptic cells sensitive to changes in presynaptic activity levels, whereas facilitation makes postsynaptic cells more responsive to bursts of presynaptic activity (Fortune and Rose, 2002; Abbott and Regehr, 2004). In addition, depression may prevent highly active inputs from dominating postsynaptic activity (Abbott et al., 1997). Thus, the short-term plasticity at a synapse could reflect the specific computational interactions between particular cell types.

Plasticity is somewhat variable from one synapse to another within a population, which could provide finer tuning of these computational characteristics. This fine-tuning would be influenced by how much plasticity varies for the multiple synapses that converge onto the same postsynaptic cell. If plasticity is uniform, then activity from synaptic inputs will be filtered similarly, whereas if it is not, then some inputs could come to dominate the cell's output.

These functional implications presume that plasticity is regulated, such as during development. Some synapses, such as the retinogeniculate synapse (Chen and Regehr, 2000) or the cerebel-

Received January 12, 2012; revised March 28, 2012; accepted April 18, 2012.

Author contributions:H.Y. and M.A.X.F. designed research; H.Y. performed research; H.Y. analyzed data; H.Y. and M.A.X.F. wrote the paper.

This work was supported by National Institutes of Health Grant R01 DC008125 (M.A.X.F.). We thankS. Chanda, A. Fischer, T. Jarsky, T. Ngodup, D. Power, T. Ruan, and Y. Yang for helpful comments and discussion during the project.

Correspondence should be addressed to Matthew A. Xu-Friedman, Department of Biological Sciences, University at Buffalo, State University of New York, Buffalo, NY 14260. E-mail: mx@buffalo.edu.

DOI:10.1523/JNEUROSCI.0167-12.2012

Copyright $\odot 2012$ the authors $\quad 0270-6474 / 12 / 327862-07 \$ 15.00 / 0$ lar mossy fiber (MF) (Wall, 2005), show small shifts in plasticity over development, whereas the calyx of Held synapse in the medial nucleus of the trapezoid body shows large changes (Taschenberger and von Gersdorff, 2000; Joshi and Wang, 2002; Taschenberger et al., 2002). However, it is not clear whether these findings indicate that plasticity is specifically regulated.

We have addressed these issues by studying excitatory synapses formed by auditory nerve (AN) fibers onto bushy cells (BCs) in the anteroventral cochlear nucleus (AVCN) (Lorente de Nó, 1981; Ryugo and Fekete, 1982; Limb and Ryugo, 2000). At this synapse, called the "endbulb of Held," the extent of depression varies considerably, which can have consequences for the transmission of information across the synapse (Yang and $\mathrm{Xu}$ Friedman, 2009). In an initial study, we found that there was similarity in plasticity between converging (C) endbulbs (Yang and Xu-Friedman, 2009). Here we address whether this similarity occurs presynaptically or postsynaptically and how it emerges during development. In addition, we surveyed excitatory synapses in the cerebellum and found similarity among converging synapses, suggesting this phenomenon may be widespread in the nervous system.

\section{Materials and Methods}

Brain slices of the cochlear nucleus or the cerebellum of CBA/CaJ mice of either sex were prepared as described previously (Xu-Friedman and Regehr, 2005; Yang and Xu-Friedman, 2008). BCs and cerebellar granule cells (GCs) were recorded in parasagittal slices, whereas Purkinje cells (PCs) and cerebellar stellate cells (SCs) were recorded in transverse slices. Recordings were made at $34^{\circ} \mathrm{C}$ in ACSF containing the following (in mM): $125 \mathrm{NaCl}, 26 \mathrm{NaHCO}_{3}, 1.25 \mathrm{NaH}_{2} \mathrm{PO}_{4}, 2.5 \mathrm{KCl}, 20$ glucose, 1 $\mathrm{MgCl}_{2}, 1.5 \mathrm{CaCl}_{2}, 4 \mathrm{Na}$-lactate, $2 \mathrm{Na}$-pyruvate, and $0.4 \mathrm{Na}$ L-ascorbate bubbled with $95 \% \mathrm{O}_{2} / 5 \% \mathrm{CO}_{2}$, pH 7.4 (310 mOsm). The voltage-clamp solution contained the following (in mM): $35 \mathrm{CsF}, 100 \mathrm{CsCl}, 10 \mathrm{EGTA}, 10$ HEPES, and 1 QX-314. Pipette resistances were 1-2 M $\Omega$ for BCs and PCs, and 2-3 M $\Omega$ for SCs and GCs, with series resistances of $12 \mathrm{M} \Omega$, compensated to $70 \%$. Cells were held at $-70 \mathrm{mV}$ (for AMPA EPSCs) or $+40 \mathrm{mV}$ (for NMDA EPSCs). BCs were distinguished from T-stellate cells in the 
AVCN by their EPSC decay kinetics and paired-pulse depression (Chanda and Xu-Friedman, 2010). Two separate stimulation isolation units (A365; World Precision Instruments) were used to stimulate separate synaptic inputs/pathways. Small glass pipettes were used that contained normal ACSF. Stimulation and recording were controlled by mafPC, run in Wavemetrics Igor. Reliable fiber isolation was confirmed by the absence of sharp drops or increases in EPSC amplitude, particularly during trains. Trials on which a stimulus failed to trigger an EPSC were discarded. Sudden increases in amplitude presumably reflected stimulation of multiple inputs, and the stimulus electrode was moved. Independence of inputs was verified using cross-stimulation experiments (i.e., stimulating input 1 then input 2 and vice versa) and confirming that each input had EPSCs of identical amplitude regardless of whether it came first or second. This technique is applicable for both depressing and facilitating synapses. The amplifier was a Multiclamp 700B (Molecular Devices). Dual simultaneous recordings were used for recordings of nonconverging (NC) inputs (see Fig. 4). Synapses were allowed $10 \mathrm{~s}$ to recover from paired stimulation and $30 \mathrm{~s}$ from train stimulation. Drugs used were strychnine (10 $\mu \mathrm{M}$, all AVCN recordings), bicuculline ( $25 \mu \mathrm{M}$, all cerebellar recordings), NBQX (10 $\mu \mathrm{M}$; see Fig. 4), MK-801 (4 $\mu$ M; see Fig. 4), and cyclothiazide (CTZ; $50 \mu$; see Fig. 3).

To quantify plasticity for a synapse, we measured EPSCs under various conditions (pairs of pulses at different intervals, trains at different frequencies). Each stimulus condition was repeated approximately five times, and the EPSCs were averaged together. EPSCs were normalized to the initial, rested EPSC of each synapse. Each normalized EPSC was considered a measure $\left(x_{i}\right)$, and the plasticity of a synapse was a collection of these measures, expressed as a vector: $\vec{x}=$ $\left(x_{1}, x_{2}, \ldots, x_{n}\right)$. To quantify the difference in plasticity between two synapses $(\vec{x}$ and $\vec{y})$, we calculated the Pythagorean distance between these vectors: $|\vec{x}-\vec{y}|=\sqrt{\sum_{i}\left(x_{i}-y_{i}\right)^{2}}$. To assess whether this similarity was significant for two converging synapses, we compared against distances between nonconverging synapses using the Kolmogorov-Smirnov test for statistical significance. This metric has a number of advantages. First, it is unbiased in terms of emphasizing different EPSC measures as carrying more information about plasticity than others. Second, the distance measure treats both endbulbs symmetrically. Asymmetrical comparisons, such as simple subtraction or ratios, would introduce bias. Third, any unanticipated bias in this calculation should also impact comparisons between nonconverging endbulbs, so our statistical approach effectively removes this as a problem.

\section{Results}

We characterized plasticity by measuring normalized EPSC amplitudes for each synapse in response to pairs of pulses (Fig. 1 Bi) as well as trains of 20 stimuli at physiologically relevant AN firing rates $(100,200$, and $333 \mathrm{~Hz}$ ) (Sachs and Abbas, 1974). To compare the plasticity of two endbulbs, we summed the square of the difference between the EPSC amplitudes and took the square root (Fig. 1 Bii). We refer to this as the "distance" between the endbulbs being compared. To decide whether plasticity was similar, we compared the distance between endbulbs that converged onto the same BC against endbulbs that terminated on different BCs recorded from the same animal on the same day (Fig. $1 A$ ). This was an important refinement over our initial study (Yang and Xu-Friedman, 2009) because it avoided the potential effects of variability between preparations.

We analyzed distances for these two populations using cumulative frequency histograms and compared the distributions with the Kolmogorov-Smirnov test. Converging endbulbs were significantly more similar than nonconverging endbulbs when the distance measure included paired-pulse ratio (PPR) data (Fig. $1 C)$ as well as low-frequency trains $(100 \mathrm{~Hz}, 20$ pulses) (Fig. $1 D)$. When the distance measure only included data from higher frequency trains, converging synapses were not significantly more similar (Fig. 1E,F), perhaps because stochastic aspects of neu-
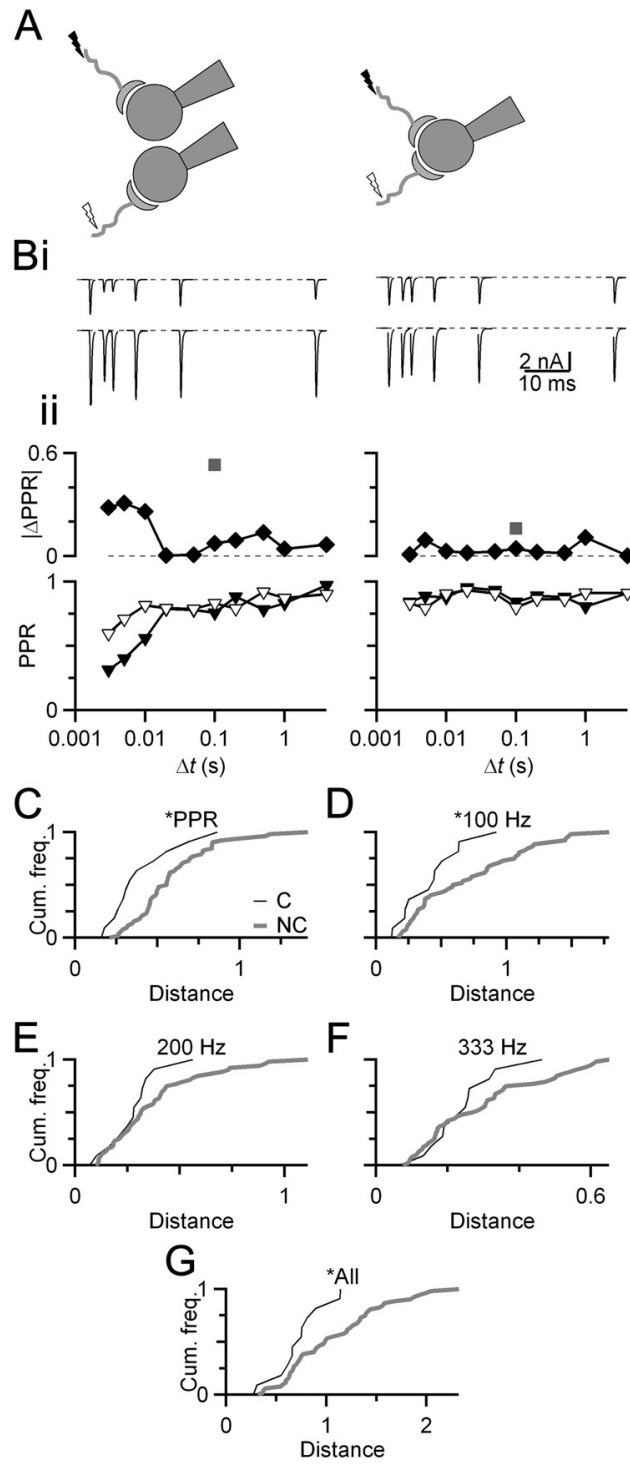

Figure 1. Converging inputs are similar. $A$, Diagram of experimental arrangement. EPSCS were recorded for $\mathrm{AN}$ fiber synapses that converge on the same $\mathrm{BC}$ or onto different $\mathrm{BCS}$. $\boldsymbol{B}$, Representative voltage-clamp experiments of converging and nonconverging endbulb EPSCs. $B i$, EPSCs resulting from stimulating individual inputs (top vs bottom traces) at different pairedpulse intervals. Bii, Quantification of short-term plasticity using the PPR (EPSC $/$ /EPSC $)$. PPR for individual endbulbs is shown by triangles (bottom). The absolute difference between these two curves is plotted and shown by diamonds (top). The square root of the sum of squares of these values is termed the distance between the endbulbs (gray squares). $\mathbf{C}-\mathbf{G}$, Cumulative frequency histograms of distances between PPR of converging (black) and nonconverging (gray) endbulbs (C), or using a similar analysis for trains of 20 stimuli at $100($ D) , 200 (E), or $333(\boldsymbol{F}) \mathrm{Hz}$. An overall distance incorporating all PPR and train measures is shown $(\boldsymbol{G})$. The distributions in $\mathbf{C}, \mathbf{D}$, and $\mathbf{G}$ are significantly different (Kolmogorov-Smirnov test, $p<0.05 ; N_{\mathrm{C}}=12$ pairs; $N_{\mathrm{NC}}=53$ pairs). Slices are from mice aged P18-P21.

rotransmitter release dominated EPSC measurements at higher frequency. When all EPSC measures were used, including PPR and trains of all frequencies, converging inputs were significantly more similar than nonconverging endbulbs (Fig. $1 G$ ). This suggested that the plasticity of multiple endbulbs forming synapses on the same BC was coordinated.

The measurements of similarity above considered EPSC amplitudes normalized to the initial rested EPSC amplitude $\left(\right.$ EPSC $\left._{1}\right)$, which emphasized similarity in the probability of release $\left(P_{\mathrm{r}}\right)$. We also considered whether other aspects of neu- 
rotransmitter release were similar by considering EPSC $_{1}$ without normalization. There was no apparent similarity in the absolute EPSC $_{1}$ amplitude of converging inputs (Fig. 2A). The relative amplitude of the smaller of two converging inputs varied from 20 to $100 \%$ of the larger (Fig. 2 B). The distribution showed two modes, one near $100 \%$ and one near $40 \%$, with an overall average of $55 \pm 5 \%\left(N_{\mathrm{C}}=27\right.$ pairs $)$. Relative amplitudes of the two converging inputs seemed to be unrelated to absolute EPSC $_{1}$ amplitude because this was found with both large and small EPSCs (Fig. 2C, flat lines). This suggests that the two modes visible in Figure $2 B$ do not reflect fundamentally different cell types. Furthermore, these results indicate that plasticity, but not absolute EPSC amplitude, is coordinated, suggesting that this coordination relates to the computational characteristics of the synapse.

We wanted to know where the similarity in plasticity was expressed. Endbulb depression results from presynaptic and postsynaptic mechanisms (Oleskevich et al., 2000). Postsynaptic plasticity results from AMPAR desensitization (Isaacson and Walmsley, 1996). This seems like a simple mechanism of coordinating converging inputs by, for example, a given $\mathrm{BC}$ expressing AMPAR subtypes with similar desensitization kinetics at all synapses. However, desensitization only affects plasticity over short time intervals ( $\sim 20 \mathrm{~ms}$ ) (Yang and Xu-Friedman, 2008; Chanda and Xu-Friedman, 2010), so it could only account for a small part of coordinated plasticity. To test this possibility, we used CTZ, which specifically affects desensitization at the endbulb (Yang and Xu-Friedman, 2008; Chanda and Xu-Friedman, 2010). CTZ has the greatest effect on PPR over

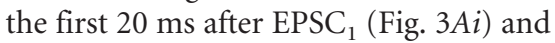
also has its greatest effect early in trains. The remaining depression after applying CTZ (the CTZ-insensitive component) probably reflects a presynaptic form of depression. We hypothesized that if presynaptic characteristics were similar for converging endbulbs (e.g., $P_{\mathrm{r}}$ ), the CTZinsensitive component of depression should be similar. In addition, if postsynaptic characteristics were similar for converging endbulbs, then the CTZ-sensitive component of depression should be similar. These two possibilities are not mutually exclusive.

We examined the CTZ-sensitive component by taking the difference before and after CTZ application for PPR or trains data (Fig. 3Aii). We found that the distances between converging endbulbs for this CTZ-sensitive component were significantly lower than for nonconverging endbulbs (Fig. 3C). This indicated that the postsynaptic component of depression was similar for converging inputs. This finding could also be influenced by presynaptic characteristics because the amount of desensitization depends on the amount of glutamate release and on postsynaptic characteristics such as receptor kinetics. We first tested this by analyzing the CTZ-insensitive component by comparing PPR and trains in the presence of CTZ (Fig. $3 A i$, closed symbols). In this experiment, converging inputs were not more similar than nonconverging inputs (Fig. 3B). However,
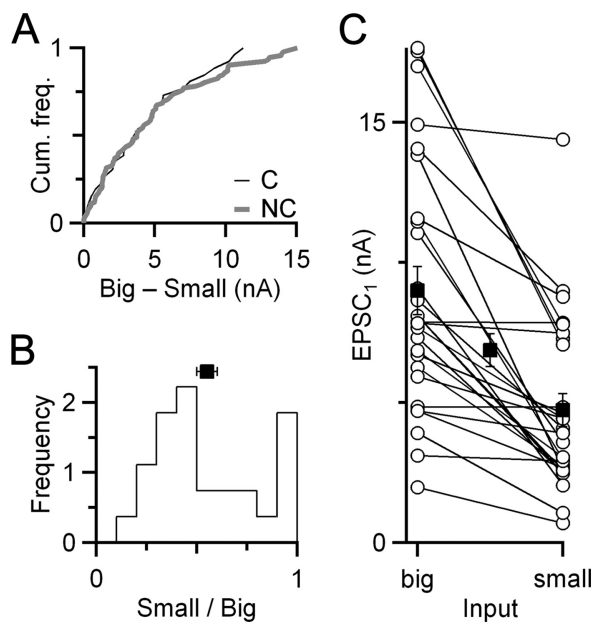

Figure 2. Coordinated plasticity is not reflected in similarity in $E P S C_{1} . A$, Cumulative frequency histogram of difference in EPSC for converging versus nonconverging inputs. There is no significant difference ( $p>0.95$, Kolmogorov-Smirnov test; $N_{\mathrm{C}}=27$ pairs, $N_{\mathrm{NC}}=93$ pairs). $\boldsymbol{B}$, Relative amplitudes of converging endbulbs ( $N_{\mathrm{C}}=27$ pairs). The relatively smaller input is scaled to the larger one. The population average is indicated by the square (mean $\pm S E M$ ). $C$, Relationships between the absolute EPSC ${ }_{1}$ amplitudes for converging endbulbs. Open circles indicate amplitudes of individual endbulbs. Converging endbulbs are connected by lines. Closed squares indicate average amplitudes of relatively larger and smaller endbulbs in the pair and the overall average (middle square).
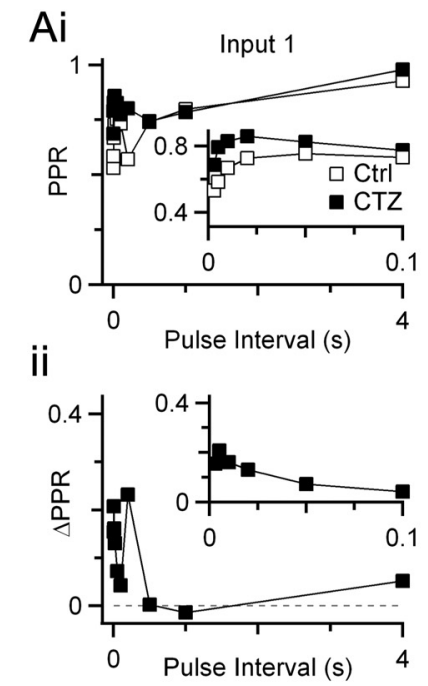

Figure 3. Effects of CTZ on similarity of converging endbulbs. Ai, Representative experiment of two converging inputs (left and right) before and after treatment with $50 \mu \mathrm{M}$ CTZ. Aii, Change in PPR ( $\triangle \mathrm{PPR})$ resulting from CTZ for the two endbulbs. Insets show PPR and $\triangle P P R$ at short intervals. $B$, Cumulative histogram of plasticity distances for converging and nonconverging endbulbs in the presence of CTZ (solid symbols in $\mathbf{A i}$ ). The distance measure incorporates PPR as well as EPSC amplitudes from the initial seven pulses during 100, 200, and $333 \mathrm{~Hz}$ trains when desensitization is significant (Chanda and Xu-Friedman, 2010) (i.e., Distance $=\sqrt{\left.\Sigma\left(\text { EPSC }_{\mathrm{CTZ}}^{\mathrm{AN} 1}-\text { EPSC }_{\mathrm{CTZ}}^{\mathrm{AN} 2}\right)^{2}\right)}$. The difference in distributions is not significant (Kolmogorov-Smirnov test, $p=$ $0.16 ; N_{\mathrm{C}}=11$ pairs, $N_{\mathrm{NC}}=24$ pairs). C, Cumulative frequency plot of the CTZ-sensitive component of plasticity. Responses in CTZ were subtracted from control (as in Aii), and distances were computed between those differences (i.e., Distance $=\sqrt{\left.\Sigma\left(\Delta \mathrm{EPSC}_{\mathrm{AN} 1}-\Delta \mathrm{EPSC}_{\mathrm{AN} 2}\right)^{2}\right)}$. Converging endbulbs have significantly lower distances than nonconverging endbulbs (Kolmogorov-Smirnov test, $p<0.05 ; N_{\mathrm{C}}=11$ pairs, $N_{\mathrm{NC}}=24$ pairs). Slices are from mice aged P16-P21. considering that the trend was in the direction of similarity, we tried a second approach to test for presynaptic similarity.

To better address whether the presynaptic aspects of synaptic transmission are similar between converging endbulbs, we compared $P_{\mathrm{r}}$ between endbulbs using the use-dependent blocker MK-801 (Hessler et al., 1993; Rosenmund et al., 1993). For nonconverging inputs, two BCs were recorded at the same 

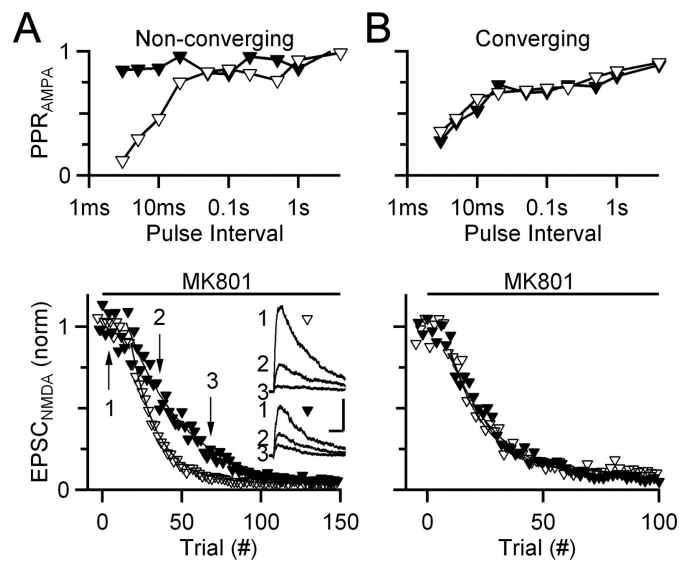

C

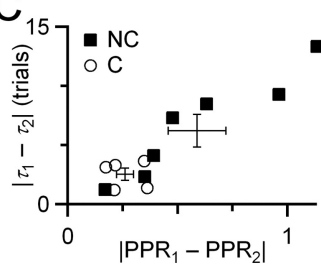

Figure 4. Experiments using MK-801 indicate that converging endbulbs have a similar probability of presynaptic neurotransmitter release. $\boldsymbol{A}, \boldsymbol{B}$, Representative experiments showing the effects of MK-801 on converging and nonconverging endbulbs. Top, PPR curves for two endbulbs that end on two simultaneously recorded $\mathrm{BCs}(\boldsymbol{A})$ or a single $\mathrm{BC}(\boldsymbol{B})$ held at $-70 \mathrm{mV}$. Bottom, Effects of MK-801 on NMDA receptor-mediated EPSCs for nonconverging $(\boldsymbol{A})$ or converging $(\boldsymbol{B})$ endbulbs. To measure NMDA EPSCs, BCs were held at $+40 \mathrm{mV}$ in the presence of NBQX. Inputs were stimulated alternately every $10 \mathrm{~s}$. Arrows indicate the timing of the NMDA EPSCS shown in the inset. Lines are exponential fits to the data used for measuring the $\tau$ of decay. Inset scale bars are $10 \mathrm{~ms}$ and $0.5 \mathrm{nA}$. C, Correlation between similarity in PPR and in decay of NMDA EPSC. The abscissa is the distance between PPR curves (as in Fig. 1C). The ordinate is the difference in the $\tau_{\text {decay }}$ from experiments similar to $\boldsymbol{A}$ and $\boldsymbol{B}$. Converging endbulbs cluster at low distance values, whereas nonconverging endbulbs are more widely distributed in both measures. Crosses show the population averages, which are significantly different $\left(p<0.05, t\right.$ test; $N_{\mathrm{C}}=5$ pairs, $N_{\mathrm{NC}}=7$ pairs). Slices are from mice aged P16-P21.

time to ensure the drug conditions were identical (Fig. 4A). One $\mathrm{AN}$ input onto each BC was isolated, and then the plasticity of AMPAR-mediated EPSCs was characterized. It was only practical to assess plasticity using PPR measurements because of the limited duration of the experiment (Fig. 4A, top). Then the NMDA receptor-mediated EPSC was measured at a holding potential of $+40 \mathrm{mV}$ in NBQX (Fig. $4 \mathrm{~A}$, bottom, insets). Once a stable baseline was recorded, MK-801 was applied, and each input was stimulated alternatively every $10 \mathrm{~s}$ (Fig. $4 A$, bottom). MK-801 was applied until the NMDA EPSC became very reduced, and the decay of peak EPSC amplitude over trials was fit to an exponential function. A faster $\tau$ of decay indicates a higher $P_{\mathrm{r}}$. For converging inputs, a single BC was recorded, and two AN inputs were isolated before AMPAR and NMDA receptor-mediated EPSCs were measured, similar to what was done in the previous experiment (Fig. $4 B$ ).

We compared simultaneously recorded endbulbs by considering the plasticity distance $\left(\left|\mathrm{PPR}_{1}-\mathrm{PPR}_{2}\right|\right)$ and the difference in $\tau_{\text {decay }}$. Converging endbulbs had both similar PPR plasticity and similar $\tau_{\text {decay }}$ (Fig. $4 C$, open circles). By contrast, nonconverging endbulbs had a wide range of PPR distances and a wide range of differences in $\tau_{\text {decay }}$ (Fig. $4 C$, closed squares). For nonconverging endbulbs, the difference in $\tau_{\text {decay }}$ in MK-801 correlated strongly with the difference in AMPA PPR, further suggesting a specific relationship of each with $P_{\mathrm{r}}$. The overall averages for converging and nonconverging endbulbs were significantly different (Fig.
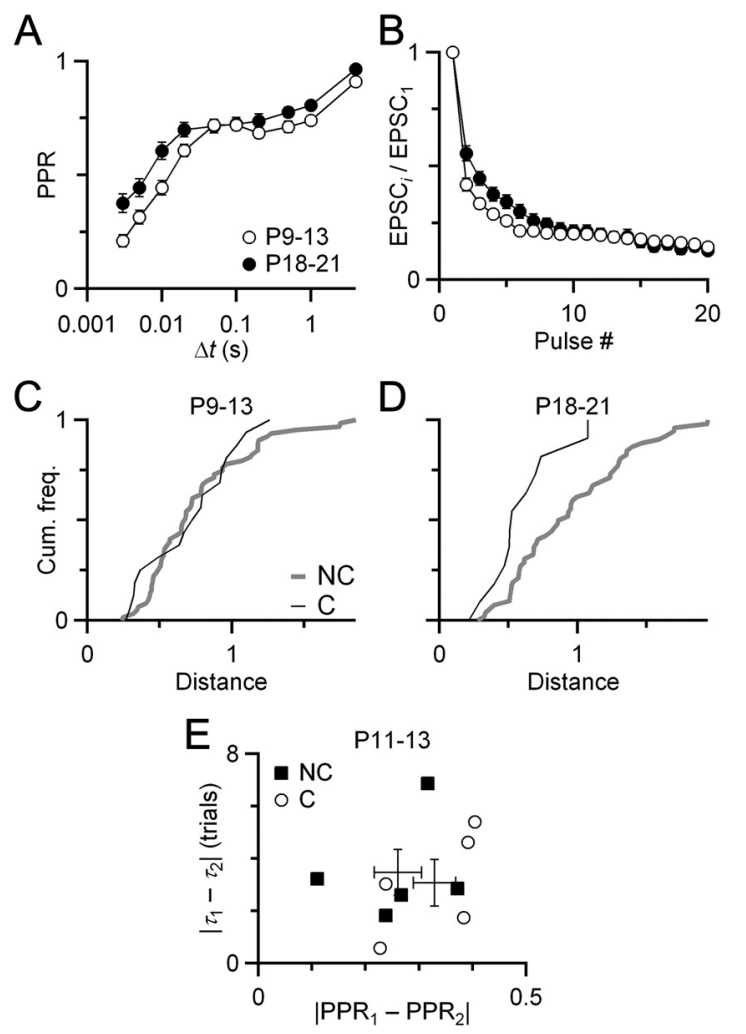

Figure 5. Coordinated plasticity emerges after the onset of hearing. $A, B$, Plasticity changes over development, with a small decrease in depression seen both for pairs of pulses $(A)$ and during $100 \mathrm{~Hz}$ trains $(\boldsymbol{B})$. Data for P9-P13 are from 17 endbulbs, and data for P18 -P21 are from 12 endbulbs. C, Cumulative frequency plot of distance for converging and nonconverging endbulbs before the onset of hearing. The two curves are not significantly different (KolmogorovSmirnovtest, $p>0.5 ; N_{\mathrm{C}}=17$ pairs, $N_{\mathrm{NC}}=60$ pairs). The distance metric includes PPR and 100 $\mathrm{Hz}$ train measurements. $\boldsymbol{D}$, For comparison, we show the same distance metric for endbulbs after the onset of hearing, which is significantly different $\left(p<0.04 ; N_{\mathrm{C}}=12\right.$ pairs, $N_{\mathrm{NC}}=53$ pairs). $\boldsymbol{E}$, MK-801 experiment similar to that of Figure 4 but in juvenile endbulbs. The converging and nonconverging populations overlap considerably, indicating no particular similarity of endbulbs before the onset of hearing ( $p>0.28, t$ test; $N_{\mathrm{C}}=5$ pairs, $N_{\mathrm{NC}}=5$ pairs).

$4 C$, crosses). These results indicated that converging endbulbs have similar presynaptic $P_{\mathrm{r}}$.

An alternative explanation of these results could be that converging synapses have similar rates of decay in MK-801 because of glutamate spillover. One prediction of this is that if glutamate spilled between adjacent synapses, the $\tau_{\text {decay }}$ for converging synapses should be faster than for nonconverging synapses, which are distant and are activated only at every other stimulus trial. However, the rates of decay in MK-801 for converging $\left(\tau_{\text {decay }}=\right.$ $10.8 \pm 0.8$ trials; $n=10)$ and nonconverging $\left(\tau_{\text {decay }}=12.1 \pm 1.3\right.$ trials; $n=14)$ endbulbs are identical ( $p>0.2$, unpaired onetailed $t$ test). Thus, spillover does not appear to influence the assessment of $P_{\mathrm{r}}$ using MK-801. A second potential issue is NMDA receptor saturation, which does occur at the endbulb (Yang and Xu-Friedman, 2008). Saturation is only partial; NMDA EPSCs during extended trains show significant temporal summation and are much larger than EPSCs after single stimuli (Pliss et al., 2009). Partial saturation could cause the relationship between $P_{\mathrm{r}}$ and $\tau_{\text {decay }}$ to differ from linearity, which would affect comparisons between both nonconverging and converging synapses. However, converging endbulbs had similar $\tau_{\text {decay }}$ in MK801, whereas nonconverging endbulbs did not. Furthermore, these differences correlated with differences in plasticity. There- 


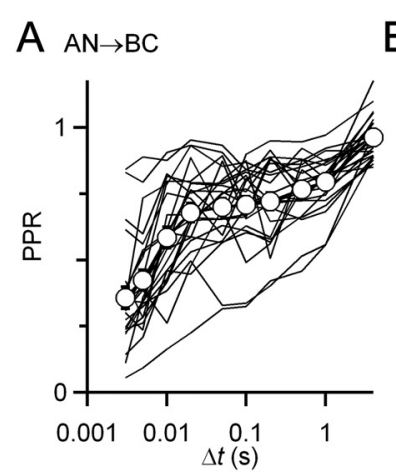

$\mathrm{B} P \mathrm{PF} \rightarrow \mathrm{PC}$
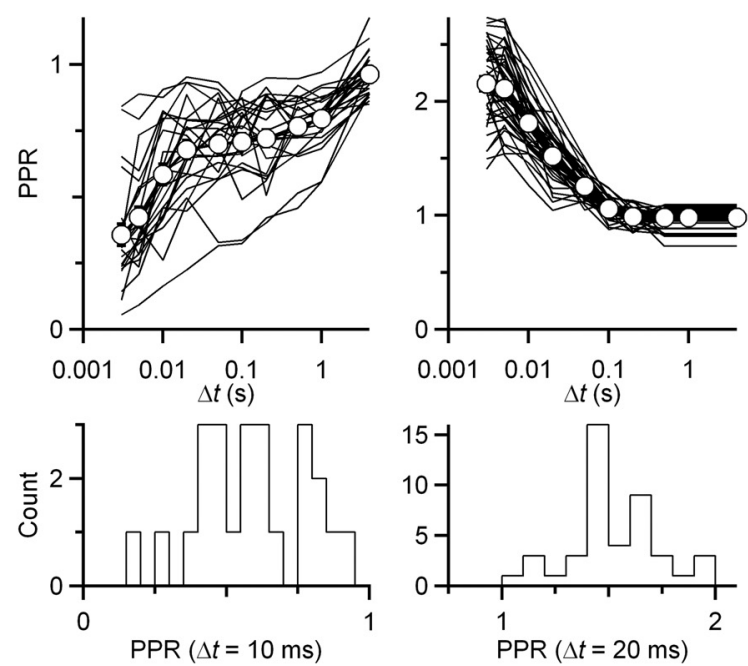

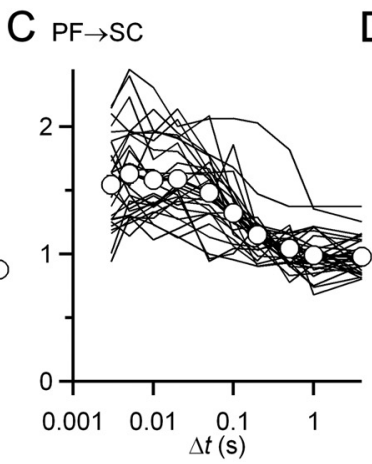

$\mathrm{D} M \mathrm{MF} \rightarrow \mathrm{GC}$
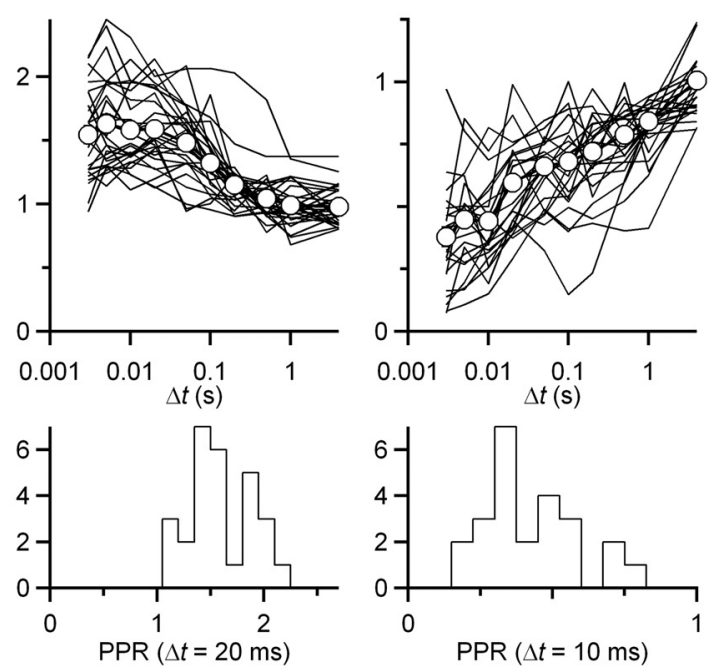

Figure 6. Plasticity in each population of recorded cells is unimodally distributed. $\boldsymbol{A}-\boldsymbol{D}$, Top, Paired pulse recovery curves for all characterized synapses $[A N$ to $B C(A N \rightarrow B C)(A)$, mossy fiber to granule cell $(\mathrm{MF} \rightarrow \mathrm{GC})(\boldsymbol{D})$ ] or pathways $(\mathrm{PF} \rightarrow \mathrm{PC})(\boldsymbol{B})$, parallel fiber to stellate cell $(\mathrm{PF} \rightarrow \mathrm{SC})(\boldsymbol{C})$ ]. Each line represents a separate synapse or pathway. Open circles and bold lines are population averages. There is considerable variability in plasticity across each population. Bottom, Representative histograms at individual time intervals when plasticity is particularly strong. No measure shows multiple modes, suggesting that each group of recordings is drawn from an effectively uniform population.

fore, partial NMDA receptor saturation does not prevent the relative assessment of $P_{\mathrm{r}}$ using MK-801.

To begin to understand how similarity is established, we examined when it emerges developmentally. We measured plasticity of converging and non-converging endbulbs in mice before the onset of hearing (i.e., before P14). Stimulation of these young synapses at frequencies $\geq 200 \mathrm{~Hz}$ was somewhat less reliable, so we restricted the distance measure to PPR and $100 \mathrm{~Hz}$ train EPSCs (Fig. 5A). The distribution of distances between converging endbulbs overlapped with the distances between nonconverging endbulbs, showing no significant difference (Fig. 5B). Before hearing onset, there was no difference between the overall mean converging and nonconverging distances, but after the onset of hearing, the nonconverging endbulbs were significantly more different than the converging endbulbs (Fig. $5 C, D$ ), as was seen with the larger metric in Figure $1 G$. This could not have resulted from the overall change in plasticity in Figure $5 B$, because nonconverging endbulbs are also maturing, yet are less similar than converging endbulbs. We confirmed this using the MK-801 method of Figure 4. Juvenile converging and nonconverging endbulbs had similar differences in both PPR and $\tau_{\text {decay }}(p>0.28, t$ test; $N_{\mathrm{C}}=5$ pairs, $N_{\mathrm{NC}}=5$ pairs) (Fig. $5 E$ ). There was an overall shift in plasticity over development (Fig. $5 A$ ), which might have reflected an overall decrease in release probability. If this accounted for the coordination phenomenon, the nonconverging synapses should also have remained similar. In fact, they did not: converging synapses are more similar than nonconverging synapses. Thus, it cannot be a nonspecific effect of changes in the population during aging.

One possible explanation for these results is that these recordings are made from at least two separate populations of cells that have distinct plasticity. In other words, it is possible that the coordinated plasticity we observed really reflected target cellspecific synaptic plasticity. There are multiple types of BCs that, within cats, have clearly defined differences in morphology and projection patterns (Osen, 1969; Cant and Morest, 1979). Recently, two groups of BCs have been distinguished in mice, but their plasticity appears identical (Cao and Oertel, 2010). We ver- ified whether we could detect multiple subtypes by examining PPR for all endbulbs in the population studied. We saw no clusters of subgroups of endbulbs either in the entire PPR curve (Fig. $6 \mathrm{~A}$, top) or for any interval plotted individually (Fig. $6 \mathrm{~A}$, bottom). Thus, it appears that these recordings were from a uniform population of endbulbs.

We evaluated whether coordinated plasticity may represent a more general phenomenon by considering excitatory synapses in the cerebellum. Cerebellar synapses also undergo significant changes in young animals, maturing somewhat later than auditory synapses. We made recordings from PCs, SCs, and GCs. We could isolate and stimulate individual MF synapses onto GCs, but this was not practical for parallel fiber (PF) synapses onto PCs or SCs. Instead, we reasoned that if individual PFs were similar, then groups of PFs should also be similar, so we stimulated larger assemblies of PFs in the molecular layer and assessed their similarities. We focused on PPR data for the distance metric. Similar to the analysis of endbulbs (Fig. 6A), each synapse appeared to be drawn from a largely uniform population of synapses (Fig. $6 B-D)$.

We found that converging synapses in the cerebellum also showed similarity. The distances in plasticity between PF synapses onto the same PC were smaller than the distances for synapses onto different PCs (Fig. 7A). For PF synapses onto SCs in the molecular layer, this was not significant in slices from younger animals (P16-18, $p>0.05)$, but it was significant in slices from older animals (P22, $p<0.04)$ (Fig. 7B). MF synapses onto GCs showed a similar developmental effect: converging inputs were not particularly similar in slices from younger animals $(\mathrm{P} 14-17, p>0.2)$ but were similar in older animals (P23-26, $p<$ 0.02 ) (Fig. $7 C$ ). This shift took place at an older age in the cerebellum than in the cochlear nucleus (i.e., P14) (Fig. 5). These results suggest that coordinated plasticity could be a common feature among converging synapses in the nervous system. Furthermore, they suggest that the coordination mechanism does not require axosomatic synapses such as endbulbs or synapses in direct proximity but can occur when synapses are located on disparate parts of the dendritic arbor. 
A $\mathrm{PF} \rightarrow \mathrm{PC}$

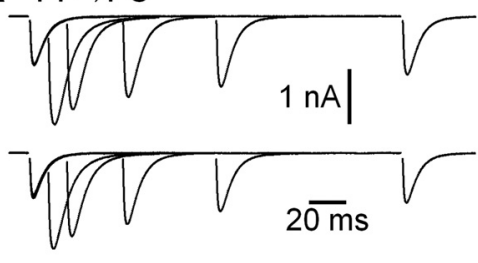

B $\mathrm{PF} \rightarrow \mathrm{SC}$
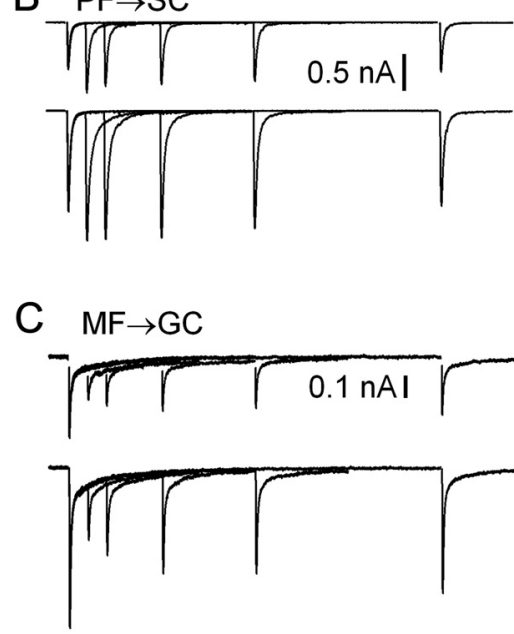
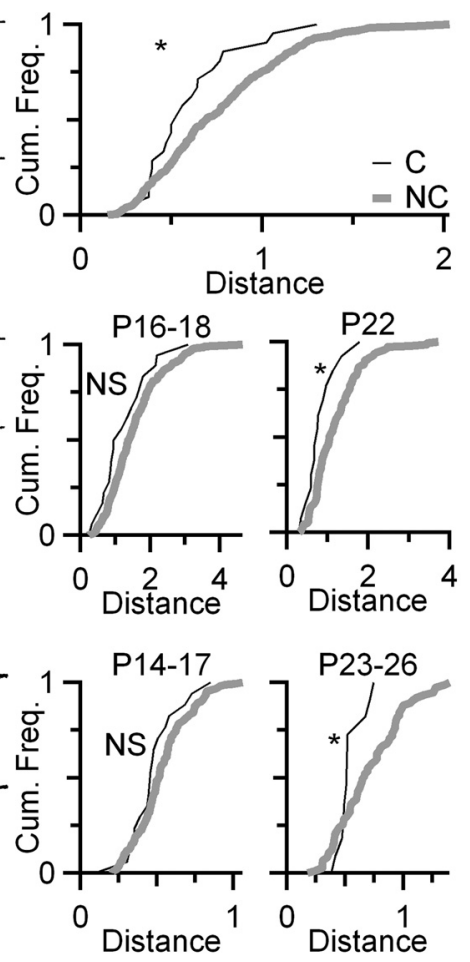

Figure 7. $\mathbf{A}-\boldsymbol{C}$, Synapses in the cerebellum show similarity in converging inputs. Representative traces from individual pathways or synapses are shown on the left. Comparisons between converging and nonconverging synapses are shown on the right. The synapses considered were $\mathrm{PF} \rightarrow \mathrm{PC}(\boldsymbol{A}), \mathrm{PF} \rightarrow \mathrm{SC}(\boldsymbol{B})$, and $\mathrm{MF} \rightarrow \mathrm{GC}(\boldsymbol{C})$. Coordinated plasticity for MF $\rightarrow \mathrm{GC}$ and PF $\rightarrow \mathrm{SC}$ terminals was significant only in older synapses. The Kolmogorov-Smirnov statistical results are as follows: PF $\rightarrow$ PC: P16-P19, $p<0.04, N_{\mathrm{C}}=22$ pairs, $N_{\mathrm{NC}}=348$ pairs $(\boldsymbol{A}) ; \mathrm{PF} \rightarrow \mathrm{SC}: \mathrm{P} 16-\mathrm{P} 18, p>0.05, N_{\mathrm{C}}=19$ pairs, $N_{\mathrm{NC}}=348$ pairs, and P22 (three preparations), $p<0.03, N_{\mathrm{C}}=14$ pairs, $N_{\mathrm{NC}}=144$ pairs $(\boldsymbol{B}) ; \mathrm{MF} \rightarrow \mathrm{GC}: \mathrm{P} 14-\mathrm{P} 17, p>0.2, N_{\mathrm{C}}=18$ pairs, $N_{\mathrm{NC}}=152$ pairs, and P23-P26, $p<0.02, N_{C}=12$ pairs, $N_{C}=76$ pairs $(C)$.

\section{Discussion}

We show here a previously unknown form of synaptic regulation in which synaptic inputs that converged on the same postsynaptic cell had similar short-term plasticity. For AN fibers, this appeared to arise through a similarity in presynaptic $P_{\mathrm{r}}$. Furthermore, the similarity emerged after the onset of hearing for AN fibers and even later for cerebellar PF and MF synapses. The coordinated plasticity we observed differed from target cell-specific synaptic plasticity (Markram et al., 1998; Reyes et al., 1998; Rozov et al., 2001), which has been described comparing synapses onto different target cell types. We observed regulation on the scale of individual cells. This could confer distinct computational properties to individual neurons, effectively yielding a diverse population at the functional level.

Our findings raise questions about how plasticity becomes coordinated. The converging synapses have the postsynaptic cell in common, yet, at least for endbulbs, it is the presynaptic $P_{\mathrm{r}}$ that is similar (Fig. 4). This could involve some retrograde signal from the common postsynaptic cell instructing the presynaptic side to adjust $P_{\mathrm{r}}$. Alternatively, it could be that the presynaptic fibers share some other characteristic that correlates with $P_{\mathrm{r}}$. One possibility is the tonotopic origin in the cochlea, but this is unlikely because plasticity shows no organization along the tonotopic axis in the AVCN. Another possibility is that AN fibers with similar spontaneous firing rates converge on the same cell (Ryugo and Sento, 1991). This could have accounted for the coordination we observed, provided $P_{\mathrm{r}}$ is regulated by activity, as has been suggested in hippocampal cell culture (Branco et al., 2008). Indeed, endbulbs in deaf strains of mice appear to have different $P_{\mathrm{r}}$ from hearing strains (Oleskevich and Walmsley, 2002), so this is a plausible mechanism.

A second issue is whether the plasticity of converging inputs converges over development or whether the plasticity of nonconverging synapses diverges. The distances between nonconverging synapses appear smaller before the onset of hearing than after the onset of hearing (Fig. 5, compare $C, D$, gray lines). This is particularly striking for the MK-801 experiments in which older synapses show a wide range of differences in plasticity and $\tau_{\text {decay }}$ (compare Figs. $4 D, 5 E$ ). In other words, it may be that converging endbulbs retain similarity over development, whereas nonconverging endbulbs become more diverse. This poses the same essential problem about how endbulbs are stabilized, but it may reflect quite different computational goals.

One question is how coordinated plasticity would interact with the various forms of long-term plasticity, which are thought to underlie learning and memory. When the expression of long-term plasticity is postsynaptic (through changes in receptor number), induction would not be expected to change shortterm plasticity (Manabe and Nicoll, 1994). However, synapses that express presynaptic forms of long-term plasticity would undergo changes in $P_{\mathrm{r}}$ and shortterm plasticity. Long-term plasticity has not been described at the endbulb, but PF synapses show both presynaptic and postsynaptic mechanisms of expression (Jorntell and Hansel, 2006), and MF synapses show presynaptic mechanisms of expression (Sola et al., 2004). It is not yet clear whether these mechanisms support/ cause coordinated plasticity or interfere with it.

One issue for evaluating whether this phenomenon is present at additional synapses is the sensitivity of our approach. This was impacted by the fact that measures of short-term plasticity are inherently variable, which likely added noise to our distance measurement. Furthermore, this is essentially a phenomenon of residual variance. Plasticity is primarily set by the cell types forming the synapse (i.e., target cell-specific synaptic plasticity). What we quantified here was what remained-that is, the synapses that formed on the same cell deviated from the mean in the same way. Comparing variances requires larger sample sizes than comparing means. These detection issues compounded the technical challenges of isolating distinct axons or pathways in brain slices and may make studying other synapses challenging.

Coordinated plasticity is likely to have functional consequences. Starting EPSC amplitudes are not particularly similar in converging inputs (Fig. 2), so it appears that what is being coordinated is not the strength of the synapse per se but rather how the strength of the synapse changes with activity. This would produce synapses that filter presynaptic activity in common ways. We have investigated the effects of different degrees of depression using dynamic clamp (Yang and Xu-Friedman, 2009). The present results suggest coordinated plasticity would yield a 
diverse population of BCs sensitive to different temporal aspects of presynaptic activity.

Questions have been raised recently about the importance of plasticity to neuronal function, at least in part because spontaneous activity may reduce the importance of the rested, initial $P_{\mathrm{r}}$ (Hermann et al., 2007; Lorteije et al., 2009; Wang et al., 2010). If plasticity were unimportant, one might expect it to be random, but our results indicate that this is not the case; it is, in fact, tightly regulated. Thus, coordinated plasticity provides indirect evidence that the initial $P_{\mathrm{r}}$ plays an important functional role in the auditory pathway and the cerebellum.

We found that plasticity becomes coordinated after the onset of hearing for the endbulb and later in the cerebellum, perhaps as locomotor behavior develops. We do not yet know whether this requires activity (spontaneous or sound-driven) or simply aging. Synaptic maturation in the visual system seems highly dependent on visual experience around the time of eye opening ( $\mathrm{Lu}$ and Constantine-Paton, 2004). Homeostatic processes influence mEPSC amplitudes (Turrigiano et al., 1998) and have also been suggested to play a role in setting $P_{\mathrm{r}}$ on different branches of cultured hippocampal neurons (Branco et al., 2008). We found yet greater uniformity in our acute brain slices, in which axosomatic synapses ( $\mathrm{AN} \rightarrow \mathrm{BC}$ ) and synapses onto separate dendrites $(\mathrm{MF} \rightarrow \mathrm{GC})$ were coordinated.

\section{References}

Abbott LF, Regehr WG (2004) Synaptic computation. Nature 431:796-803.

Abbott LF, Varela JA, Sen K, Nelson SB (1997) Synaptic depression and cortical gain control. Science 275:220-224.

Branco T, Staras K, Darcy KJ, Goda Y (2008) Local dendritic activity sets release probability at hippocampal synapses. Neuron 59:475-485.

Cant NB, Morest DK (1979) Organization of the neurons in the anterior division of the anteroventral cochlear nucleus of the cat: lightmicroscopic observations. Neuroscience 4:1909-1923.

Cao XJ, Oertel D (2010) Auditory nerve fibers excite targets through synapses that vary in convergence, strength, and short-term plasticity. J Neurophysiol 104:2308-2320.

Chanda S, Xu-Friedman MA (2010) A low-affinity antagonist reveals saturation and desensitization in mature synapses in the auditory brainstem. J Neurophysiol 103:1915-1926.

Chen C, Regehr WG (2000) Developmental remodeling of the retinogeniculate synapse. Neuron 28:955-966.

Fortune ES, Rose GJ (2002) Roles for short-term synaptic plasticity in behavior. J Physiol Paris 96:539-545.

Hermann J, Pecka M, von Gersdorff H, Grothe B, Klug A (2007) Synaptic transmission at the calyx of Held under in vivo like activity levels. J Neurophysiol 98:807-820.

Hessler NA, Shirke AM, Malinow R (1993) The probability of transmitter release at a mammalian central synapse. Nature 366:569-572.

Isaacson JS, Walmsley B (1996) Amplitude and time course of spontaneous and evoked excitatory postsynaptic currents in bushy cells of the anteroventral cochlear nucleus. J Neurophysiol 76:1566-1571.

Jörntell H, Hansel C (2006) Synaptic memories upside down: bidirectional plasticity at cerebellar parallel fiber-Purkinje cell synapses. Neuron $52: 227-238$

Joshi I, Wang LY (2002) Developmental profiles of glutamate receptors and synaptic transmission at a single synapse in the mouse auditory brainstem. J Physiol 540:861-873.

Limb CJ, Ryugo DK (2000) Development of primary axosomatic endings in the anteroventral cochlear nucleus of mice. J Assoc Res Otolaryngol 1:103-119.

Lorente de Nó R (1981) The primary acoustic nuclei. New York: Raven.

Lorteije JA, Rusu SI, Kushmerick C, Borst JG (2009) Reliability and precision of the mouse calyx of Held synapse. J Neurosci 29:13770-13784.

Lu W, Constantine-Paton M (2004) Eye opening rapidly induces synaptic potentiation and refinement. Neuron 43:237-249.

Manabe T, Nicoll RA (1994) Long-term potentiation: evidence against an increase in transmitter release probability in the CA1 region of the hippocampus. Science 265:1888-1892.

Markram H, Wang Y, Tsodyks M (1998) Differential signaling via the same axon of neocortical pyramidal neurons. Proc Natl Acad Sci U S A 95:5323-5328.

Oleskevich S, Walmsley B (2002) Synaptic transmission in the auditory brainstem of normal and congenitally deaf mice. J Physiol 540:447-455.

Oleskevich S, Clements J, Walmsley B (2000) Release probability modulates short-term plasticity at a rat giant terminal. J Physiol 524:513-523.

Osen KK (1969) Cytoarchitecture of the cochlear nuclei in the cat. J Comp Neurol 136:453-484.

Pliss L, Yang H, Xu-Friedman MA (2009) Context-dependent effects of NMDA receptors on precise timing information at the endbulb of Held in the cochlear nucleus. J Neurophysiol 102:2627-2637.

Reyes A, Lujan R, Rozov A, Burnashev N, Somogyi P, Sakmann B (1998) Target-cell-specific facilitation and depression in neocortical circuits. Nat Neurosci 1:279-285.

Rosenmund C, Clements JD, Westbrook GL (1993) Nonuniform probability of glutamate release at a hippocampal synapse. Science 262:754-757.

Rozov A, Burnashev N, Sakmann B, Neher E (2001) Transmitter release modulation by intracellular $\mathrm{Ca}^{2+}$ buffers in facilitating and depressing nerve terminals of pyramidal cells in layer $2 / 3$ of the rat neocortex indicates a target cell-specific difference in presynaptic calcium dynamics. J Physiol 531:807-826.

Ryugo DK, Fekete DM (1982) Morphology of primary axosomatic endings in the anteroventral cochlear nucleus of the cat: a study of the endbulbs of Held. J Comp Neurol 210:239-257.

Ryugo DK, Sento S (1991) Synaptic connections of the auditory nerve in cats: relationship between endbulbs of Held and spherical bushy cells. J Comp Neurol 305:35-48.

Sachs MB, Abbas PJ (1974) Rate versus level functions for auditory-nerve fibers in cats: tone-burst stimuli. J Acoust Soc Am 56:1835-1847.

Sola E, Prestori F, Rossi P, Taglietti V, D’Angelo E (2004) Increased neurotransmitter release during long-term potentiation at mossy fibregranule cell synapses in rat cerebellum. J Physiol 557:843-861.

Taschenberger H, von Gersdorff H (2000) Fine-tuning an auditory synapse for speed and fidelity: developmental changes in presynaptic waveform, EPSC kinetics, and synaptic plasticity. J Neurosci 20:9162-9173.

Taschenberger H, Leão RM, Rowland KC, Spirou GA, von Gersdorff $\mathrm{H}$ (2002) Optimizing synaptic architecture and efficiency for highfrequency transmission. Neuron 36:1127-1143.

Turrigiano GG, Leslie KR, Desai NS, Rutherford LC, Nelson SB (1998) Activity-dependent scaling of quantal amplitude in neocortical neurons. Nature 391:892-896.

Wall MJ (2005) Short-term synaptic plasticity during development of rat mossy fibre to granule cell synapses. Eur J Neurosci 21:2149-2158.

Wang Y, Ren C, Manis PB (2010) Endbulb synaptic depression within the range of presynaptic spontaneous firing and its impact on the firing reliability of cochlear nucleus bushy neurons. Hear Res 270:101-109.

Xu-Friedman MA, Regehr WG (2005) Dynamic-clamp analysis of the effects of convergence on spike timing, I: many synaptic inputs. J Neurophysiol 94:2512-2525.

Yang H, Xu-Friedman MA (2008) Relative roles of different mechanisms of depression at the mouse endbulb of Held. J Neurophysiol 99:2510-2521.

Yang H, Xu-Friedman MA (2009) Impact of synaptic depression on spike timing at the endbulb of Held. J Neurophysiol 102:1699-1710. 